\title{
Multi-Resonant Class-F Power Amplifier Design for 5G Cellular Networks
}

\author{
Maryam SAJEDIN ${ }^{1,2}$, Issa ELFERGANI ${ }^{1,4}$, Jonathan RODRIGUEZ ${ }^{1,3}$, Manuel VIOLAS ${ }^{2}$, \\ Abdalfettah ASHARAA ${ }^{4}$, Raed ABD-ALHAMEED ${ }^{4}$, Monica FERNANDEZ-BARCIELA ${ }^{5}$, \\ Ahmed M. ABDULKHALEQ ${ }^{6}$
}

\author{
${ }^{1}$ Instituto de Telecomunicações, Campus Universitário de Santiago, 3810-193 Aveiro, Portugal \\ ${ }^{2}$ Departamento de Electrónica, Telecomunicações e Informática, Universidade de Aveiro, 3810-193 Aveiro, Portugal \\ ${ }^{3}$ Faculty of Computing, Engineering and Science, University of South Wales, Pontypridd CF37 1DL, UK \\ ${ }^{4}$ Faculty of Engineering and Informatics, Bradford University, Bradford BD7 1DP, UK \\ ${ }^{5}$ Department of Signal Theory and Communications, University of Vigo, 36310 Vigo, Spain \\ ${ }^{6}$ SARAS Technology Limited, Leeds LS12 4NQ, UK
}

Maryam.sajedin@av.it.pt

Submitted September 26, 2020 / Accepted April 24, 2021

\begin{abstract}
This work integrates a harmonic tuning mechanism in synergy with the GaN HEMT transistor for $5 G$ mobile transceiver applications. Following a theoretical study on the operational behavior of the Class-F power amplifier (PA), a complete amplifier design procedure is described that includes the proposed Harmonic Control Circuits for the second and third harmonics and optimum loading conditions for phase shifting of the drain current and voltage waveforms. The performance improvement provided by the Class-F configuration is validated by comparing the experimental and simulated results. The designed $10 \mathrm{~W}$ Class-F PA prototype provides a measured peak drain efficiency of $64.7 \%$ at $1 \mathrm{~dB}$ compression point of the $P A$ at $3.6 \mathrm{GHz}$ frequency.
\end{abstract}

\section{Keywords}

Power amplifiers, GaN HEMT, class-F, power dissipation, heat transfer

\section{Introduction}

The $5 \mathrm{G}$ mobile vision is expected to provide an enhanced broadband service, whilst addressing architectural changes to cater for massive scale connectivity (Internet of Things) that is driven by the vertical industries. A fully deployed 5G system will harness high-band small cell technology based on millimeter wave and advanced antenna technology, forming a tight synergy that will have a direct multiplier effect on the single link data rate performance, where design targets aim to achieve gigabit per second speeds. However, with the foreseen increase in mobile traffic, network operators also aim to reduce the cost of ownership of their networks, where operational expenses such as energy consumption represents a major cost. These requirements translate to energy efficient net- work designs that profit all the way to the RF hardware. In fact, PAs represent the largest consumer of energy in the transceiver chipset, hence positioning power efficient designs are of paramount importance.

It is well known that high efficiency can be achieved in PAs at the compressed gain levels in the presence of small conduction angles. However, very small conduction angle at higher frequencies requires greater input voltage swing for similar output current excursions, which reduces the power gain [1]. While the higher output power can be guaranteed by maximizing both the output current and voltage swing, the maximum efficiency can be attained by minimizing the dissipated power in device. The non-overlapping drain waveforms condition enables the PA to fulfil this requirement. Harmonic terminating techniques enable PAs to provide higher efficiency by selecting appropriate waveforms. The overdriven Class-F PA proposed by Snider in [2], relies on the load impedance at the fundamental and harmonic frequencies whilst, Class-E or D focus on the transistor switching mode operation [3], [4]. Harmonic engineered amplifiers, irrespective of the drain bias current and the pinching-off time of the transistor, enhance the fundamental voltage component for a sufficiently high input power level, while maintaining the DC power supplied to a similar level. In Class-F, $\mathrm{F}^{-1}$ [5], [6] and switched-mode approaches, the role of the generated harmonics is to ensure an appropriate wave-shaping. On the other hand, the restrictions imposed by the device output nonlinearities deteriorate the adverse effects of the phase imbalance on the fundamental output current and voltage waveforms.

To enhance the waveforms harmonic content, many contributions from experimental observation of the transistor operation to the introduction of innovative configurations have been proposed. In [7], the authors realized a GaAs MMIC Class-F PA at $14.3 \mathrm{GHz}$ incorporated an active phase array for higher date rate transmission. In 
that work, a Class-F PA is design in power stage and a driver stage is added to improve the large-signal gain at the expense of fabrication complexity and cost. The proposed MMIC PA can provide a drain efficiency of $50.4 \%$ and $19.7 \mathrm{~dB}$ gain. In [8], an improvement in gain and efficiency are reported by the Class-F PA connected to the active antenna, where the impedance of the antenna is optimized to provide harmonic matching for the Class-F PA. Nevertheless, the internal parasitics of packaged transistors prevent the PA to deliver its optimum performance.

Concurrently, the continuous modes of operation have been proposed for providing high efficiency across a wide frequency range, where the short and open circuited load terminations are replaced by purely reactive harmonic termination. To expand the impedance design space, a concurrent continuous Class-F PA has been adapted in [9] that can provide $72.8 \%$ and $76.7 \%$ drain efficiency at 3.5 and $2.6 \mathrm{GHz}$, respectively. However, the continuous PA modes require to control the harmonic impedances precisely for high efficiency performance and should maintain high peak voltage waveforms over the frequency range, that can be supported only by high breakdown voltage technologies like GaN devices, otherwise, it results in low output power density.

In this work an optimized design procedure to enhance the efficiency and gain of a Class-F PA over the Class-B configuration, by adopting a proposed harmonic control technique for second and third harmonics tuning is described. This technique can effectively compensate the detrimental effects of the packaged GaN HEMT parasitic components. For this purpose, the Class-F PA' output power and efficiency expressions are discussed using device time-domain waveforms. Subsequently, the PA performance is validated through the layout design, test of the fabricated prototype as well as, analysis of the simulated and measurement results.

This work is organized as follows: a brief theory of Class-F PA is elaborated in Sec. 2; Section 3 is devoted to present the design procedure of PA; this section also includes the PA implementation and measurement results. The last section outlines the conclusion of this work.

\section{Basic Operation of Class-F Power Amplifier}

PA efficiency can be improved by driving the active device into the triode or cut-off or regions, when either the current or voltage waveforms are shaped as a square wave. The flat waveform contains only odd frequency components when the harmonics are terminated with an open circuit at the intrinsic drain of the device. In the Class-F mode, this flattening is executed on the voltage waveform. Consequently, the correlated half-sinusoidal current and square-wave voltage spectra at the fundamental frequency have no RF dissipated power theoretically.
Whilst the general concept of multi-harmonic manipulation strategy is elaborated in [10], [11], Raab [12] has studied the effects of tuning a finite order of harmonics on the Class-F PA and derived the harmonic magnitudes required for flattening the voltage waveform. Nevertheless, the high-efficiency harmonic tuning techniques require to analyze the PA linearity characteristics or even enforce an external linearization circuit in multicarrier systems. In many harmonic injection PA stages, proper subsystem linearization techniques have been adopted to combat spectral regrowth of incorporated signals. For linearity enhancement, the large-signal intermodulation distortion (IMD) sweet spot is introduced in [13], as a theoretical solution to achieve low IMD in the saturation region.

The analysis assumes that the transistor is controlled by the input gate-source voltage linearly and the intrinsic transistor output response is represented by the device trans-conductance. The half-sine wave drain current is expressed by (1)

$$
i_{\mathrm{D}}(t)=I_{\mathrm{DM}} \cdot \cos \left(\omega_{0} t\right),-\pi / 2<\omega_{0} t<\pi / 2
$$

where the maximum drain current is shown by $I_{\mathrm{DM}}$. The drain current is equal to zero for $-\pi<\omega_{0} t<-\pi / 2$ and $\pi / 2<\omega_{0} t<\pi$. Considering the third harmonic components, the representation of the drain voltage waveform can be obtained by (2)

$$
v_{\mathrm{D}}(t)=V_{\mathrm{dc}}-V_{1} \cos \left(\omega_{0} t\right)+V_{3} \cos \left(3 \omega_{0} t\right)
$$

where $V_{\mathrm{dc}}$ is the DC supply voltage. The peak-to-peak swing of the ac component of drain to source voltage waveform will be decreased because of adding the out-ofphase third harmonic component to the fundamental voltage, and the maximum ac component's value of voltage waveform becomes lower than the amplitude of the fundamental component $V_{1}$. Besides, it is possible to raise the ratio $V_{1} / V_{\mathrm{dc}}$ by increasing the amplitude of the third harmonic component. When $V_{1}$ rises and the input power keeps constant, the output RF power and the drain efficiency will enhance by the same factor. This will be shown in the following analysis.

The Fourier coefficients for the maximum drain to source voltage can be obtained by setting the derivatives equal to zero. The first-order derivative is zero at the minimum value of $\omega_{0} t=0$ and the maximum value of $\omega_{0} t=\pi$. From the second-order derivative at $\omega_{0} t=0$, equation (3) can be expressed by

$$
\left.\frac{\mathrm{d}^{2} v_{\mathrm{DS}}}{\mathrm{d}\left(\omega_{0} t\right)^{2}}\right|_{\omega_{0} t=0}=V_{1} \cos \left(\omega_{0} t\right)-9 V_{3} \cos \left(3 \omega_{0} t\right)=0 .
$$

Therefore, a maximum drain voltage waveform occurs when $V_{3} / V_{1}=1 / 9$, and the minimum drain-source voltage is obtained as (4)

$$
V_{\mathrm{DSmin}}=V_{\mathrm{ds}}-V_{1}+V_{3}=V_{\mathrm{ds}}-\frac{8}{9} V_{1}=0 .
$$


That produces the maximum amplitude of the output voltage $V_{1}=9 / 8 V_{\mathrm{ds}}$, and by substituting $V_{1}$ in (4), the required amplitude of the third harmonic voltage component can be attained as $V_{3}=V_{\mathrm{ds}} / 8$.

The fundamental drain current amplitude can be expressed by

$$
I_{1}=\frac{9\left(V_{\mathrm{ds}}-V_{\mathrm{DSmin}}\right)}{8 R_{\mathrm{L}}}
$$

The DC input current and supply power are given respectively, by (6) and (7):

$$
I_{\mathrm{DC}}=\frac{1}{2 \pi} \int_{-\frac{\pi}{2}}^{\frac{\pi}{2}} I_{\mathrm{DM}} \cos \left(\omega_{0} t\right) \mathrm{d}\left(\omega_{0} t\right)=\frac{2}{\pi} I_{1}=\frac{9\left(V_{\mathrm{ds}}-V_{\mathrm{DS} m i n}\right)}{4 \pi R},
$$

$$
P_{\mathrm{DC}}=\frac{9 V_{\mathrm{ds}}\left(V_{\mathrm{ds}}-V_{\mathrm{DS} \min }\right)}{4 \pi R} .
$$

The drain-source voltage consists of the dc, fundamental and third harmonic components, and the drain current contains a dc, fundamental and second harmonic component [14], [15], therefore, the RF output power includes the fundamental component,

$$
P_{\mathrm{O}}=\frac{1}{2} \cdot \frac{I_{\mathrm{DM}}}{2} \cdot \frac{9}{8}\left(V_{\mathrm{ds}}-V_{\mathrm{DS} \min }\right)=\frac{9}{32} I_{\mathrm{DM}}\left(V_{\mathrm{ds}}-V_{\mathrm{DS} \min }\right) .
$$

Hence, the Class-F PA drain efficiency can be achieved by

$$
\eta_{\mathrm{D}}=\frac{P_{\mathrm{O}}}{P_{\mathrm{DC}}}=\frac{1}{2} \cdot \frac{\pi}{2} \cdot \frac{V_{1}}{V_{\mathrm{ds}}}=\frac{\pi V_{1}}{4 V_{\mathrm{ds}}}=\frac{9 \pi}{32}\left(1-\frac{V_{\mathrm{DS} \min }}{V_{\mathrm{ds}}}\right) .
$$

It should be noted that the amplitude of the fundamental component $V_{1}$ has been increased with the third harmonic contribution to $V_{1}=9 V_{\mathrm{ds}} / 8=1.125 V_{\mathrm{ds}} \quad$ [16], thereby, the drain efficiency is increased by the same factor of 1.125 .

\section{Circuit Design of the Class-F PA}

This section presents the design approach of $10 \mathrm{~W}$ Class-F PA based on GaN HEMT technology. The design technology is hybrid-MIC microstrip on a Rogers 4350 substrate $\left(\varepsilon_{\mathrm{r}}=3.66\right.$ and $\left.h=0.762\right)$.

\subsection{Transistor Technology and Bias Selection}

From the nonlinear HEMT model, it is known that the maximum efficiency is restricted by the output resistance of the transistor and the drain-source capacitance. The inherent high bandgap of Gallium Nitride $(\mathrm{GaN})$ transistor offers the possibility of operating at high voltages, minimizes the mismatch losses, and improves the thermal conductivity. Since, the high breakdown voltage provides more

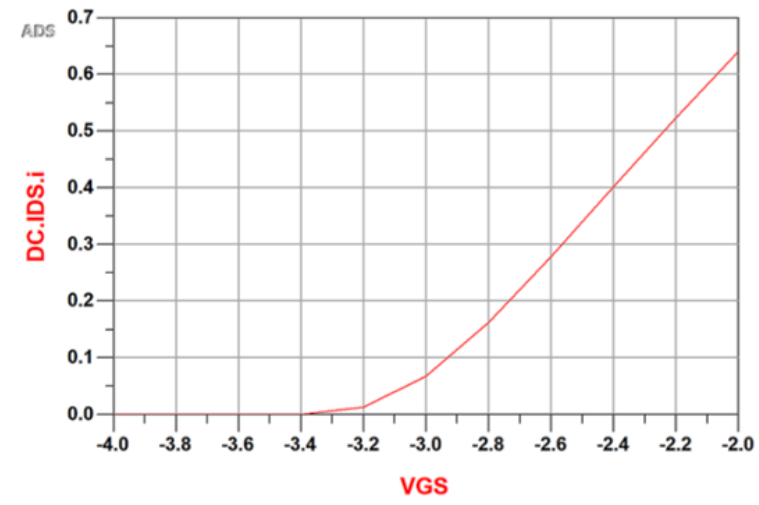

Fig. 1. $I_{\mathrm{dS}}-V_{\mathrm{GS}}$ transfer characteristic.

robust devices, GaN HEMTs on Silicon (Si) can maximize the power density and supply up to $180 \mathrm{~W}$ output RF power at the base stations [17].

By moving further towards a fully digital radio in $5 \mathrm{G}$ and beyond, GaN HEMT MMIC PAs at X-band can provide up to $55 \% \mathrm{PAE}$ and $40 \mathrm{~W}$ output power [18], [19]. In this paper, the unmatched $10 \mathrm{~W}$ GaN HEMT device provided by Cree-Wolfspeed is employed.

The packaged GaN HEMT equivalent circuit, and specifically, the CGH40010F model, includes both intrinsic and package parasitics. The presence of parasitic wiring and nonlinear variation of $V_{\mathrm{gs}}$ with respect to the input voltage, pose severe implications for harmonic terminations. Figure 1 shows the $I_{\mathrm{dS}}-V_{\mathrm{GS}}$ transfer characteristic for a fixed $V_{\mathrm{ds}}$ of $28 \mathrm{~V}$ supply voltage. Note that selecting an appropriate PA conduction angle is important since, a bias point close to the threshold gate voltage increases the harmonic component. The selected bias point must ensure the third harmonic current is null. Here a bias point slightly lower than Class-B conduction angle at $V_{\mathrm{G}}=-3.1 \mathrm{~V}$ is selected, while the lower bias points impose higher nonlinear operation regimes and provide in-phase third harmonic component.

\subsection{The Rollet Stability Factor of the PA}

The PA must be stable at all frequencies to prevent unwanted oscillations, in or out of the operating frequency band. The transistor nonlinear input capacitance exhibits negative resistance under a strong input power drive that results in a parametric generation of harmonic components [20]. The small-signal stability analysis of a PA mostly relies on the stability circles and Rollet Stability factor (K-factor), hence spurious power dependent intrinsic oscillation phenomena cannot be predicted by small-signal stability analysis. The geometric-derived stability factor from the s-parameters can assure the stability that is expressed by:

$$
\mu=\frac{1-\left|S_{11}\right|^{2}}{\left|S_{22}-\Delta S_{11}^{*}\right|+\left|S_{12} S_{21}\right|}>1 .
$$

The determinant of the scattering matrix is expressed by $\Delta$. 


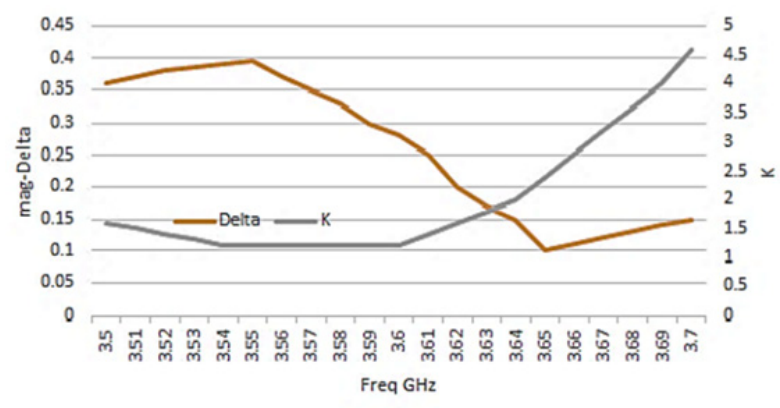

(a)

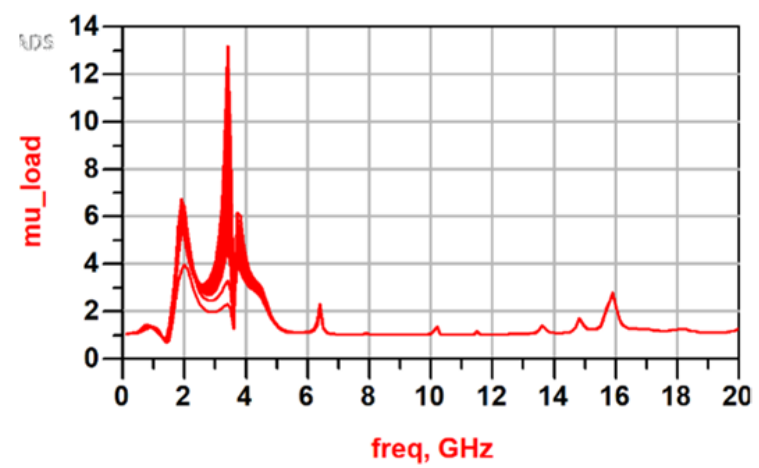

(b)

Fig. 2. Stability factors: (a) $K, \Delta$. (b) The geometrically derived load.

Observation of start-up analysis of either $K>1$ and $|\Delta|<1$, plotted in Fig. 2(a), will not guarantee unconditional stability, when the PA is terminated with an impedance having positive or zero resistances. Besides, the single geometric-derived parameter, $\mu>1$ shown in Fig. 2(b) guarantees the stability auxiliary condition. The work [22] provides the complementary large-signal stability-analysis techniques assisted to ensure the stability. By increasing the real part of impedance, the stability will be improved with the penalty of gain reduction at the operating frequency. In this study, a series resistor at the input path of the transistor improves the output Return Loss.

\subsection{Bias Network Consideration}

Design of the gate/drain bias network includes some trade-offs among linearity, stability, and complexity. A proper gate bias line provides positive input resistance for achieving low-frequency stability [23], [24]. Here, the desired bias-tees are synthesized first by decoupling capacitors to null the RF signal at the operating frequency. Then, a proper thickness of the $(\lambda / 4)$ line provides a high impedance at the fundamental frequency. The minimum $\lambda / 4$ line's width of $0.5 \mathrm{~mm}$ with characteristic impedance of $80 \Omega$ can handle the maximum $0.6 \mathrm{~A}$ drain current for the selected substrate.

\subsection{Design of Harmonic Tuning Networks}

To minimize the parasitic effects of device, the impedance terminations of fundamental and harmonics are required to provide a perfect match. In this respect, the load-pull simulations are conducted through the varying load impedance values defined by a certain VSWR and variable phase. Here, the load-pull simulations of stabilized FET transistor are run for selecting the optimal output impedance that compromise between the maximum efficiency and maximum output RF power.

The optimum I-gen impedances at the drain of transistor for the third harmonic order are shown in Fig. 3. The third harmonic is terminated close to the open circuit condition. The harmonic control of the Class-F PA is carried out by a short circuited second harmonic load, while the third harmonic is selected to maximize the output power. To present appropriate fundamental and harmonic terminations, the output matching network (OMN) is designed and optimized that includes a third-harmonic peaking circuit using a resonant tank in series with the output.

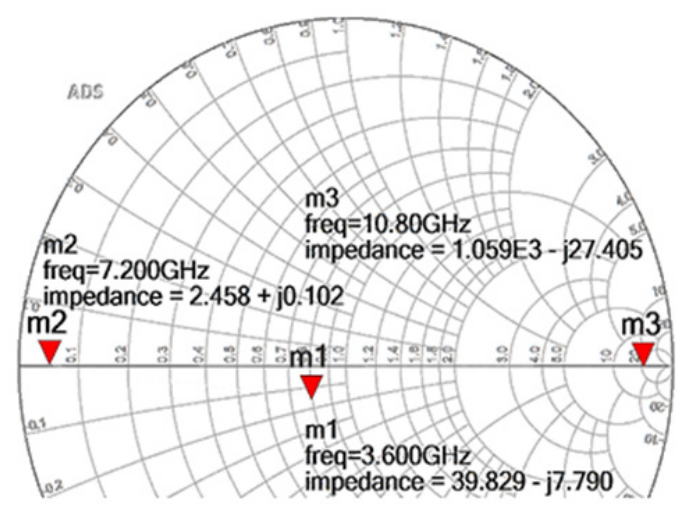

Fig. 3. S11 of the fundamental impedance, second and third harmonics at I-gen plane.

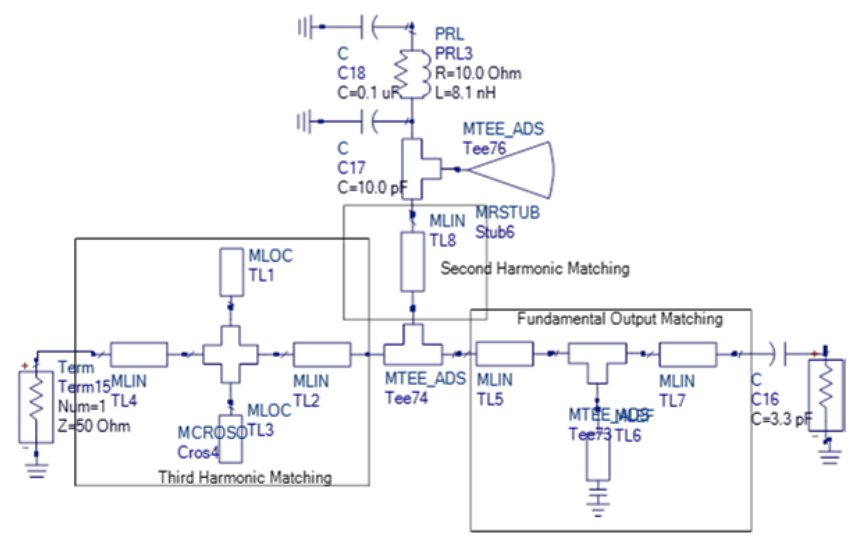

(a)

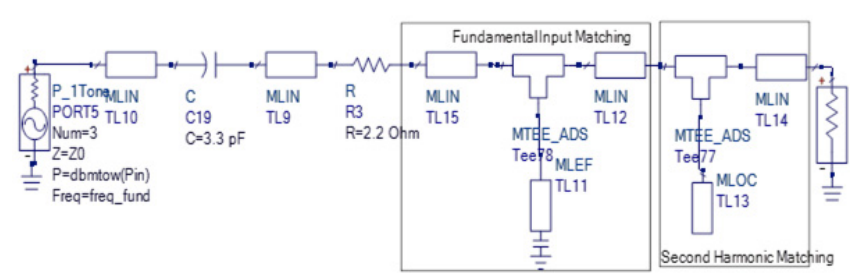

(b)

Fig. 4. Schematic of the harmonic tuning networks incorporated to (a) OMN, (b) IMN 


\begin{tabular}{|c|c|c|}
\hline Freq. & $Z_{\text {Load }}$ & $Z_{\text {in }}$ \\
\hline$F_{0}$ & $14.7+\mathrm{j} \cdot 13.8$ & $4.2-\mathrm{j} \cdot 20$ \\
\hline $2 F_{0}$ & $-\mathrm{j} \cdot 18$ & $0.01+\mathrm{j} \cdot 0.09$ \\
\hline $3 F_{0}$ & $\mathrm{j} \cdot 98$ & $0.4-\mathrm{j} \cdot 110$ \\
\hline
\end{tabular}

Tab. 1. Input and output impedances extracted by load-pull simulation.

The third harmonic voltage has a ratio of 19 to the fundamental voltage, as mentioned in Sec. 2, thus, the finite slope of a GaN device's knee boundary allows the third harmonic component to square-up the fundamental voltage waveform. Therefore, the voltage waveform contains a large amount of third harmonic component that pushes the fundamental voltage over the value dictated by the device physical limitations. Since, the third harmonic flattening does not affect the dissipated power, higher output power can be achieved. Figure 4(a) illustrates the topology of the OMN using the proposed compact Harmonic Control Circuit (HCC). The electrical length of the transmission lines in the matching networks are optimized for the Rogers 4350 substrate. A T-shape transmission line submatching network transfers the fundamental optimum load of $14.7+\mathrm{j} \cdot 13.8$ to the $50 \Omega$ and delivers the maximum excursions of the drain waveforms.

To further improve the PAE and gain, the load-pull simulations are also conducted for second and third harmonics. The harmonic impedances are listed in Tab. 1. At the third harmonic frequency, the load impedance is a large value of purely imaginary while the real part of harmonic impedance is too small to be ignored. The resonant circuit includes two open stub lines and two transmission lines connected by a junction. The electrical lengths of lines are tuned to control the harmonic terminations. To generate a proper drain current waveform, the $\lambda / 4$ line (TL8) in bias network provides a very low impedance at the second harmonic that can eliminate the distortion in the input voltage.

The input matching network (IMN) is designed to maximize the gain level at the operating frequency. The purpose of harmonic tuning at the IMN shown in Fig. 4(b), is to prevent the delivery of harmonic power to the load. Moreover, the input second harmonic impedance should be controlled as it affects the input nonlinearity. To deliver the maximum power transfer from the input source to the FET, the transistor's input is conjugate matched at the fundamental frequency. Input impedance $\left(Z_{\text {in }}\right)$ of transistor at $3.6 \mathrm{GHz}$ is $4.2-\mathrm{j} \cdot 20$ and the IMN transfers the $50 \Omega$ to the conjugate match of $Z_{\text {in }}$. Note that the HCCs are placed in front of the fundamental matching networks to prevent harmonic impedance variation. Figure 5. indicates the drain waveforms of the designed Class-F PA. Moreover, the nonlinear characteristics of PA without predistortion linearization are plotted in Fig. 6, where a slight compression at high power levels can be observed.

As a final remark, electromagnetic simulations should be performed for estimating actual measurement results, by considering the sensitivity of the transmission lines tolerances and layout disposition. The schematic circuits of the $10 \mathrm{~W}$ Class-F, including HCCs at input/output, and a single-

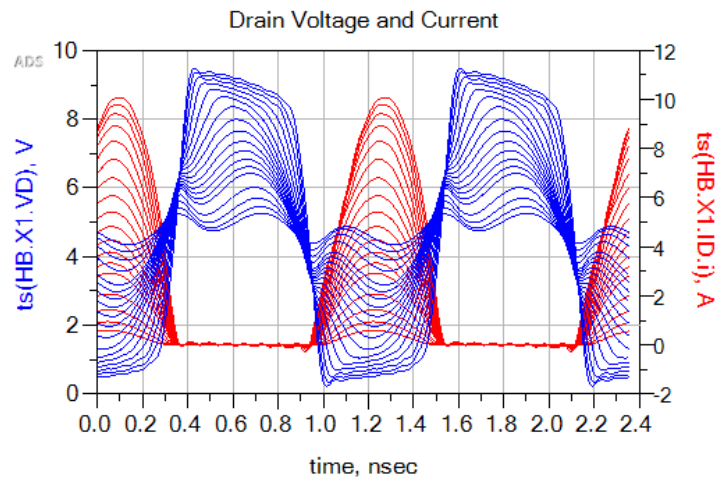

Fig. 5. Drain time-domain waveforms.

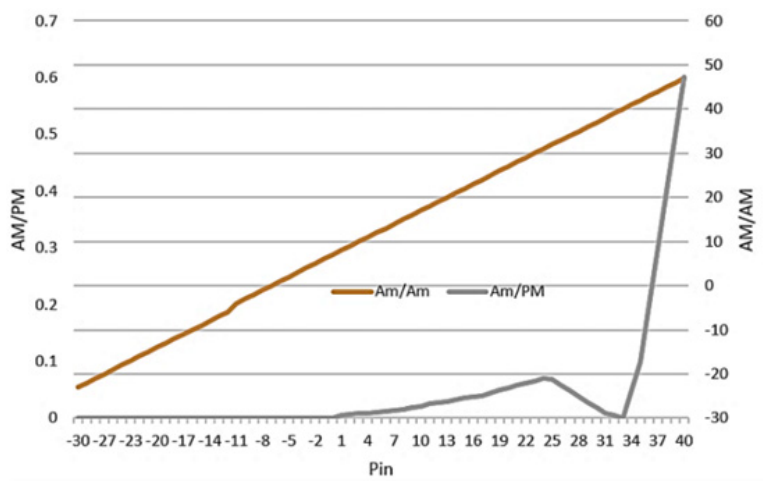

Fig. 6. AM/PM and AM/AM PA characteristics without linearization.

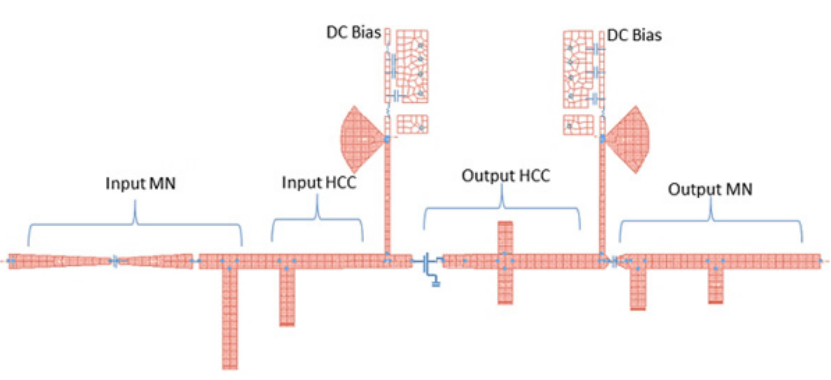

Fig. 7. Layout of Class-F PA mode.

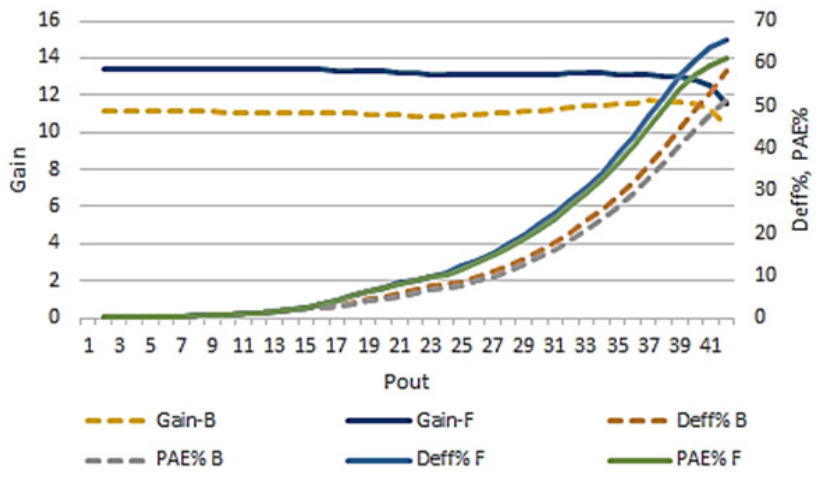

Fig. 8. Simulated gain, $P A E$ and drain efficiency of Class-F vs. those of Class-B PA.

ended Class-B PA using the same transistor and bias condition have been designed at $3.6 \mathrm{GHz}$. The layout of the designed Class-F PA is depicted in Fig. 7 where the ClassB PA is extended with harmonic resonators to shape the output voltage waveform. 
To show the effect of the harmonic voltage, the layout results of transducer power gain, drain efficiency and PAE of the Class-F PA are compared with those of the Class-B PA in Fig. 8. Dashed lines are allocated to the performance of Class-B PA. The results reveal that while both classes provide approximately the same saturated output power, Class-F PA delivers about 13\% higher PAE where the dissipation in transistor is minimized. Therefore, the harmonic tuning contributes in higher efficiency. The Class-B PA provides linear amplification by setting a perfect shortcircuit at all relevant harmonics, hence the instantaneous efficiency varies linearly with the output voltage. Besides, the Class-F PA has superior drain efficiency performance of $66 \%$ and gain flatness of $13.7 \mathrm{~dB}$ over the Class-B PA, with $56 \%$ drain efficiency and transducer gain of $11.3 \mathrm{~dB}$.

\subsection{PA Prototype Implementation and Experimental Results}

We fabricated the corresponding Class-F PA prototype for $3.6 \mathrm{GHz}$ operating frequency, to evaluate the inherent operation of the wave-form engineering. Figure 9(a) depicts the photo of the assembly mounted on an aluminum plate and the PCB dimensions. The blocking capacitors models of the ATC 800-A series are used. These capacitors provide heat transfer for high RF power levels.

Figure 9(b) exhibits the employed setup for PA measurement and test. It consists of an RF power driver (Mini-

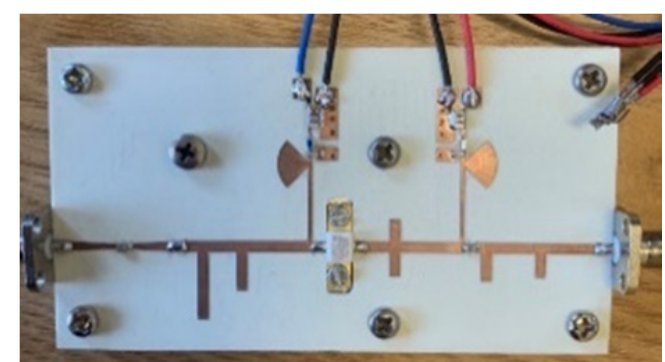

(a)

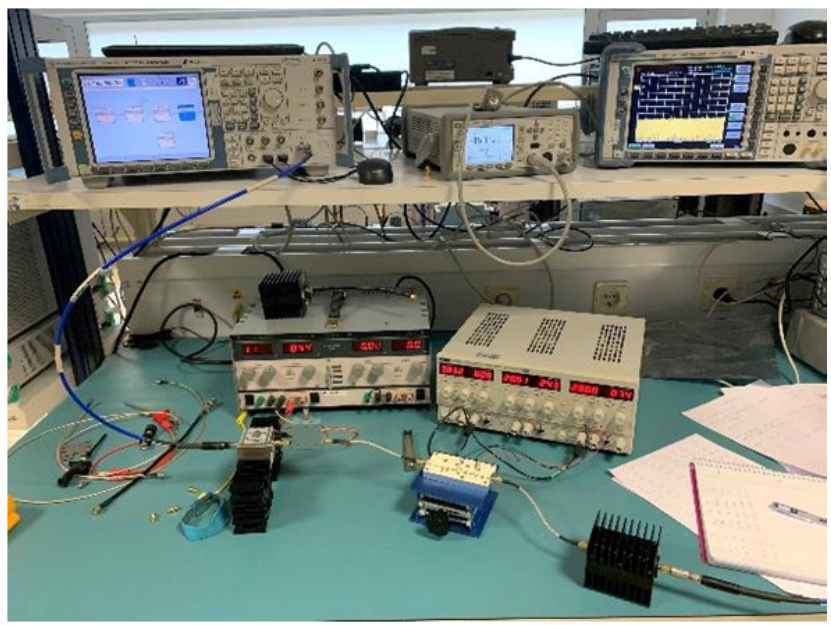

(b)

Fig. 9. (a) The PCB prototype of the Class-F PA with the dimension of $86 \mathrm{~mm} \times 31 \mathrm{~mm}$. (b) Measurement testbench for the PA.

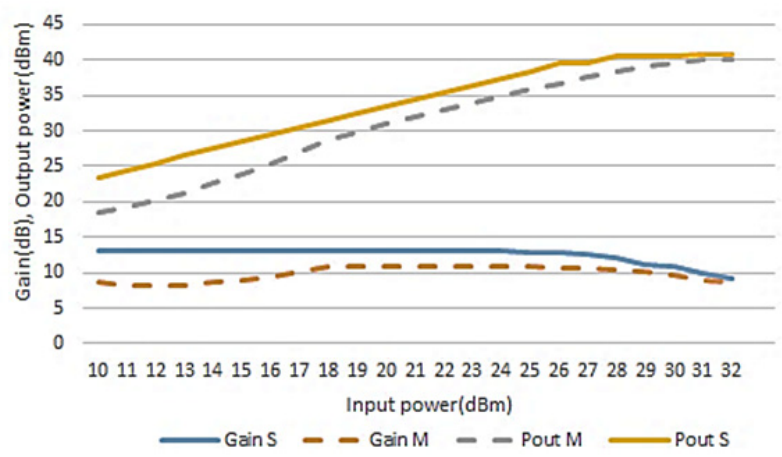

(a)

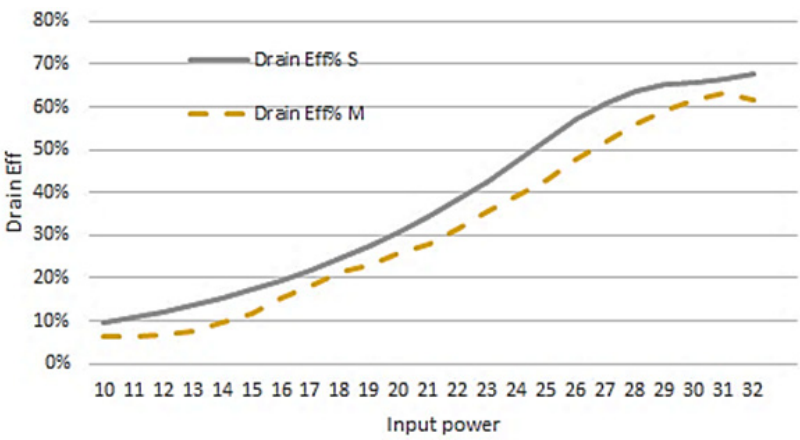

(b)



(c)

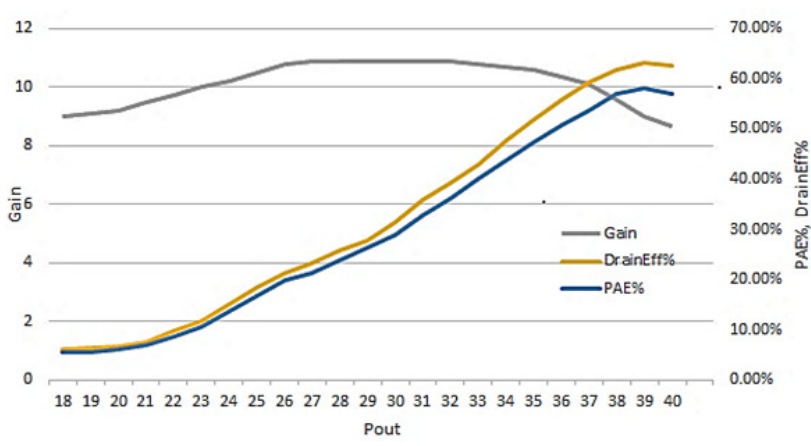

(d)

Fig. 10. Experimental results of (a) output power and gain,
(b) drain efficiency, (c) transducer power gain,
(d) PAE vs. Pout.

Circuit ZVE-8G+) to generate the input signal, and the Spectrum Analyser (SMW200A) to measure the output RF signal. A calibrated $30 \mathrm{~dB}$ fixed attenuator (BW$30 \mathrm{~N} 100 \mathrm{~W}+$ ) is used as a protective element to prevent the VSA to be overloaded at high power levels. The PA was tested with power-swept continuous wave (CW) excitations from a vector signal generator (R\&SFSW8) to study the PA behavior under different excitation levels. The funda- 
mental voltage has been increased with the input power drive; hence the current is saturated.

The large-signal performance of the PA with $28 \mathrm{~V} V_{\mathrm{ds}}$ and quiescent drain current of $15 \mathrm{~mA}$ is shown in Fig. 10 . The measured results (dashed lines) are compared to the simulated ones (solid lines).

As depicted in Fig. 10(a), (b) the PA provides a measured peak drain efficiency of $64.7 \%$ at $40 \mathrm{dBm}$ output power, while it operates at its $1 \mathrm{~dB}$ compression point. Figure 10(c) plots the small-signal gain variation through a frequency sweep from 3.4 to $3.7 \mathrm{GHz}$. The transducer power gain at $3.56 \mathrm{GHz}$ is approximately close to the simulation result, even though at $3.6 \mathrm{GHz}$ is lower than the simulation predicts. A maximum measured transducer power gain of $11 \mathrm{~dB}$ is achieved showing a $2 \mathrm{~dB}$ difference with respect to the simulation results. For higher power levels, this difference decreases. Measurement results depict the effectiveness of employed HCC when the peak PAE of $59.6 \%$ has been achieved at maximum RF output power whilst, the IMN improves the input reflection coefficient and the gain response versus frequency. Figure 10(d) depicts the measured results of PAE, drain efficiency and gain versus output power. The results indicate the experimental measurements are well-matched with the simulated results, considering the fabrication tolerances.

The obtained results are compared favorably with recently published papers on state-of-the-art Class-F PAs, listed in Tab. 2. The results confirmed that the design methodology proposed in this paper can deliver high efficiency and high linear performance without using predistortion technique. The harmonic distortion of $-52.51 \mathrm{dBc}$ for the second harmonic and $-37.41 \mathrm{dBc}$ for the third harmonic, in the measured output power spectrum, validated the results. In summary, wave-shaping matching design methodologies improve the power amplifier large-signal performance in conjunction with advanced device technologies, providing solutions suitable for $5 \mathrm{G}$ transmitters.

\section{Conclusion}

This work contributes to experimentally validate the harmonic tuning influence on the efficiency improvement of a Class-F PA, based on GaN HEMT technology for 5G

\begin{tabular}{|c|c|c|c|c|c|}
\hline Refs. & $\begin{array}{c}\text { Freq. } \\
\mathbf{G H z}\end{array}$ & $\begin{array}{c}\mathbf{P A E} \\
\mathbf{\%}\end{array}$ & $\begin{array}{c}\boldsymbol{P}_{\text {out }} \\
\mathbf{d B m}\end{array}$ & Device & $\begin{array}{c}\text { Gain } \\
\mathbf{d B}\end{array}$ \\
\hline$[\mathbf{9}]$ & $0.55-0.95$ & $70-86$ & 40 & GaN HEMT & 15 \\
\hline$[\mathbf{2 5}]$ & 5.7 & 68 & 34 & GaN HEMT & 9.5 \\
\hline$[\mathbf{2 6}]$ & 2.5 & 50 & 40 & GaN HEMT & 14 \\
\hline$[\mathbf{2 7}]$ & 5 & 58 & 28 & $\begin{array}{c}\text { GaAs } \\
\text { pHEMT }\end{array}$ & 13.5 \\
\hline$[\mathbf{2 8}]$ & $1.45-2.45$ & $60-65$ & $40.41-42$ & GaN HEMT & $10-12.6$ \\
\hline $\begin{array}{c}\text { This } \\
\text { work }\end{array}$ & 3.6 & 59.6 & 40 & GaN HEMT & 11.2 \\
\hline
\end{tabular}

Tab. 2. Performance comparison summary of Class-F PAs. applications. The designed amplifier complies with the design rules required for the quiescent point, second and third harmonic terminations. The bias network is shaped by the desired trade-off between RF isolation, current-handling and low-frequency stability. The harmonic content of the output current and voltage waveforms, shaped by appropriate terminations, minimize the overlapping region and hence the amount of dissipated power. The measured results of hybrid MIC microstrip technology are coherent with the simulated results.

\section{Acknowledgments}

This work is supported by the POSITION-II project funded by the ECSEL joint Undertaking under grant number Ecsel-7831132-Postitio-II-2017-IA, www. position2.eu, and the Spanish National Board of Scientific and Technology Research and the European Union (ERDF), through Project TEC2017-88242-C3-2-R.

\section{References}

[1] RODRIGUEZ, J., RADWAN, A., BARBOSA, C., et al. SECRET-Secure Network Coding for Reduced Energy next generation mobile small cells: A European Training network in wireless communications and networking for 5G. In 2017 Internet Technologies and Applications (ITA). Wrexham (UK), 2017, p. 329-333. DOI: 10.1109/ITECHA.2017.8101964

[2] SNIDER, D. M. A theoretical analysis and experimental confirmation of the optimally loaded and overdriven RF power amplifiers. IEEE Transactions on Electron Devices, 1967, vol. 14, no. 12, p. 851-857. DOI: $10.1109 /$ T-ED.1967.16120

[3] CRIPPS, S. C. RF Power Amplifiers for Wireless Communications. London (UK): Artech House, 2006. ISBN: 0890069891, 9780890069899

[4] CRIPPS, S. C., TASKER, P. J., CLARKE, A. L., et al. On the continuity of high efficiency modes in linear RF power amplifiers. IEEE Microwave and Wireless Component Letters, 2009, vol. 19, no. 10, p. 665-667. DOI: 10.1109/LMWC.2009.2029754

[5] COlANTONIO, P., GARCIA, J. A., GIANNINI, F., et al. High efficiency and high linearity power amplifier design. International Journal of RF and Microwave Computer Aided Engineering, 2005, vol. 15 , no. 5, p. $453-468$. DOI: $10.1002 /$ mmce. 20111

[6] LEE, Y., LEE, M., JEONG, Y. High-efficiency class-F GaN HEMT amplifier with simple parasitic-compensation circuit. IEEE Microwave and Wireless Component Letters, 2008, vol. 18, no. 1, p. 55-57. DOI: 10.1109/LMWC.2007.912023

[7] DHAR, S. K., SHARMA, T., ZHU, N., et al. Design methodology of broadband continuous class-F power amplifiers for sub-6-GHz 5G applications. IEEE Transactions on Microwave Theory and Techniques, 2020 , vol. 68 , no. 7 , p. 3120-3133. DOI: 10.1109/TMTT.2020.2984603

[8] WREN, M., BRAZIL, T. J. Experimental class-F power amplifier design using computationally efficient and accurate large-signal pHEMT model. Transactions on Microwave Theory and Techniques, 2005, vol. 53, no. 5, p. 1723-1731. DOI: 10.1109/TMTT.2005.847108

[9] OZALAS, M. T. High efficiency class-F MMIC power amplifiers at Ku-band. In The 2005 IEEE Annual Conference Wireless and 
Microwave Technology. Clearwater Beach (FL, USA), 2005, p. 24-28. DOI: 10.1109/WAMIC.2005.1528402

[10] YANG, Z., YAO, Y., LI, M., et al. A precise harmonic control technique for high efficiency concurrent dual-band continuous class-F power amplifier. IEEE Access, 2018, vol. 6, p. 51864 to 51874. DOI: 10.1109/ACCESS.2018.2870865

[11] SAJEDIN, M., ELFERGANI, I., RODRIGUEZ, J., et al. Energy efficient and wideband class-J Doherty power amplifier. In 2020 12th International Symposium on Communication Systems, Networks and Digital Signal Processing (CSNDSP). Porto (Portugal), 2020, p. 1-6. DOI: 10.1109/CSNDSP49049.2020.9249642

[12] COLANTONIO, P., GIANNINI, F., LEUZZI, G., et al. Multiharmonic manipulation for highly efficient microwave power amplifiers. International Journal of RF and Microwave Computer Aided Engineering, 2001, vol. 11, no. 6, p. 366-384. DOI: 10.1002/mmce. 1045

[13] RAAB, F. H. Class-f power amplifiers with maximally flat waveforms. IEEE Transactions on Microwave Theory and Techniques, 1997, vol. 45, no. 11, p. 2007-2012. DOI: $10.1109 / 22.644215$

[14] PEDro, J. C., CARVAlho, N. B., FAGER, C., et al. Linearity versus efficiency in mobile handset power amplifiers: A battle without a loser. Microwave Engineering Europe, 2004. p. 19-26.

[15] COLANTONIO, P., GIANNINI, F., LEUZZI, G., et al. On the class-F power amplifier design. International Journal of $R F$ and Microwave Computer Aided Engineering, 1999, vol. 9, no. 2, p. 129-149. DOI: 10.1002/(SICI)1099047X(199903)9:2<129::AID-MMCE7>3.0.CO;2-U

[16] KAZIMIERCZUK, M. RF Power Amplifier. New York (USA): Wiley, 2008. Ch. 8, p. 267-289. ISBN: 9781118844373

[17] SAJEDIN. M., ELFERGANI, I., RODRIGUEZ, J., et al. A survey on RF and microwave Doherty power amplifier for mobile handset applications. Electronics, 2019, vol. 8, no. 6, p. 1-31. DOI: 10.3390/electronics 8060717

[18] BAHL, I., BHARTIA, P. Microwave Solid State Circuit Design. Canada: John Wiley \& Sons. 2003. ISBN: 978-0-471-20755-9

[19] SAJEDIN, M., ELFERGANI, I., RODRIGUEZ, J., et al. Advancement of a highly efficient class-F power amplifier for $5 \mathrm{G}$ Doherty architectures. In 2019 IEEE 2nd 5G World Forum $(5 G W F)$. Dresden (Germany), 2019, p. 86-90. DOI: 10.1109/5GWF.2019.8911678

[20] GIANNINI, F., LEUZZI, G. Nonlinear Microwave Circuit Design. UK: John Wiley \& Sons, 2004. ISBN: 978-0-470-84701-5

[21] CABRAL, P. M., PEDRO, J. C., CARVALHO, B. Nonlinear device model of microwave power GaN HEMTs for high poweramplifier design. IEEE Transactions on Microwave Theory and Techiques, 2004, vol. 52, no. 11, p. 2585-2592. DOI: 10.1109/TMTT.2004.837196

[22] ABDUlKhALEQ, A. M., YAHYA, M. A., MCEWAN, N., et al. Recent developments of dual-band Doherty power amplifiers for upcoming mobile communications systems. Electronics, 2019, vol. 8 , no. 6 , p. 1-19. DOI: 10.3390/electronics 8060638

[23] DU, X., HELAOUI, M., YOU, C. J., et al. Analytical design space of power amplifiers including the class-A/B/J continuum for dynamic load modulation. IEEE Access, 2019, vol. 7, p. 71933-71942. DOI: 10.1109/ACCESS.2019.2919379

[24] MOON, J., JEE, S., KIM, J., et al. Behaviors of class-F and class$\mathrm{F}^{-1}$ amplifiers. IEEE Transactions on Microwave Theory and Techniques, 2012, vol. 60, no. 6, p. 1937-1951. DOI: 10.1109/TMTT.2012.2190749

[25] AGGRAWAL, E., RAWAT, K., ROBLIN, P. Investigating continuous class-F power amplifier using nonlinear embedding model. IEEE Microwave and Wireless Components Letters, 2017, vol. 27, no. 6, p. 593-595. DOI: 10.1109/LMWC.2017.2701316

[26] KURODA, K., ISHIKAWA, R., HONJO, K. High-efficiency GaN-HEMT class-F amplifier operating at $5.7 \mathrm{GHz}$. In 2008 38th European Microwave Conference. Amsterdam (Netherlands), 2008, p. 440-443. DOI: 10.1109/EUMC.2008.4751483

[27] NICKANDISH, G., BABAKRPUR, E., MEDI, A. A harmonic termination technique for single- and multi band high-efficiency class-F MMIC power amplifiers. IEEE Transactions on Microwave Theory and Techniques, 2014, vol. 62, no. 5, p. 1212-1220. DOI: 10.1109/TMTT.2014.2315591

[28] TUFFY, N., GUAN, L., ZHU, A., et al. A simplified broadband design methodology for linearized high-efficiency continuous class-F power amplifiers, IEEE Transactions on Microwave Theory and Techniques, 2012, vol. 60, no. 6, p. 1952-1963. DOI: 10.1109/TMTT.2012.2187534

\section{About the Authors}

Maryam SAJEDIN is a Ph.D. student in Electrical Engineering at the Universidade de Aveiro, Portugal since 2017. She is currently employed on the collaborative European Training Network (ETN) SECRET project, targeting research on $5 \mathrm{G}$ RF front end. She received the M.Sc degree in Electrical Engineering in 2015 and her working experiences are RF and Microwave Power Amplifiers, nonlinear modeling of high power amplifiers and compensation techniques for nonlinear distortion in OFDM system. She is a researcher at the Instituto de Telecomunicações (IT) Aveiro, Universidade de Aveiro, and she has been involved in several research projects.

Issa ELFERGANI received his M.Sc. and Ph.D. in Electronic and Electrical Engineering from the University of Bradford (UK) in 2008 and 2012, with a specialization in tunable antenna design for mobile handset and UWB applications. He is now a senior researcher at the Instituto de Telecomunicações, Aveiro (Portugal), working with European research-funded projects, while leading technical activities in antenna design for ENIAC ARTEMOS (20112014), EUREKA BENEFIC (2014-2017), CORTIF (20142017), GREEN-T (2011-2014), VALUE (2016-2016), H2020-ITN-SECRET (2017-2020) and THINGS2DO (2014-2018). He is a member of the IEEE, IET and American Association for Science and Technology (AASCIT). Issa is the Guest editor/special issue of Electronics "Recent Technical Developments in Energy-Efficient 5G Mobile Cells (ISSN 2079-9292), Special Issue on Recent Advances in Engineering Systems Journal (ASTESJ) 2017/2019 and Guest Editor/special issue of Electronics "Recent Advances in Antenna Design for 5G Heterogeneous Networks" (ISSN 2079-9292). Issa has around 135 high-impact publications in international conferences, journal papers, and book/book chapters with a Google Scholar h-index 17. He is currently conducting an excellent work towards the $5 \mathrm{G}$ single and MIMO antennas, including the physical human interaction, and links to health concern.

Jonathan RODRIGUEZ received his Master's degree in Electronic and Electrical Engineering and Ph.D. from the 
University of Surrey (UK), in 1998 and 2004 respectively. $\mathrm{He}$ is a SMIEEE, FIET, CENG and SFHEA. In 2005, he became a researcher at the Instituto de Telecomunicações (Portugal) and Senior Researcher in 2008 where he established the Mobile System Research Group. He has served as project coordinator for major international research projects, including FP7-C2POWER, H2020-ITN-SECRET, CELTIC LOOP and GREEN-T projects, while acting as Technical manager for the FP7-COGEU and FP7-SALUS, among others. In 2017 he was appointed Professor of Mobile Communications at the University of South Wales (UK). He is author of more than 600 scientific works, including 11 book editorials.

Manuel VIOLAS obtained a degree in Electronics and Telecommunications at the University of Aveiro, in 1982. He obtained a master's degree at the Faculty of Sciences of the University of Coimbra in 1988 in the specialty of Telecommunications and completed his Ph.D. in Electronic Engineering from the University of Aveiro in 1999. Since 1982 he has been teaching at the Department of Electronics Telecommunications and Informatics at the University of Aveiro. From 1990 to 1992, he developed research on high-bandwidth optical receivers at British Telecom. He is a senior researcher at the Instituto de Telecomunicações, Aveiro, Portugal having been involved in several national and international research projects in the field of optical communications. The main research interests include the design of circuits for microwaves and millimeter waves, characterization modeling and simulation of electro-optical devices, processing of electrical signals for optical communication systems with high transmission rates. More recently he has been involved in baseband signal processing algorithms implementation in FPGA. In this domain, he has been involved in the implementation of pre and post-processing algorithms for baseband signals for cooperative wireless systems and in the development of wireless system demonstrators. He published 20 articles in magazines and 32 articles at conferences.

Abdalfettah ASHARAA is currently a Ph.D. student in the Faculty of Engineering and Informatics at the University of Bradford, United Kingdom. He received his BSc. degree in Electrical and Electronic Engineering from Bani Walid University, Libya. In 2016, he received MSc. with Distinc- tion in Electrical Engineering from the University of Bradford, UK. He is author of several conference publications and his main research interests include next generation network systems, antennas and radio frequency technologies, smart grids and near field communication systems.

Raed ABD-ALHAMEED is professor of electromagnetic and radio frequency engineering at the University of Bradford, UK. He received his B.Sc. and M.Sc. degrees from Basrah University, in 1982 and 1985, respectively, and his Ph.D. degree from the University of Bradford, UK, in 1997, all in Electrical Engineering. He has many years' research experience over 25 years in the areas of radio frequency, antennas, and electromagnetic computational techniques and has published over 500 academic journal and conference papers. He is the senior academic responsible for electromagnetic research in the communications research group, in which new antenna design have been developed including several patents, which were considered and filed.

Mónica FERNANDEZ BARCIELA received the MSc degree in (Electronics) Physics in 1989 (Univ. Santiago C., Spain), and the Ph.D. degree in Telecom. Eng. (Univ. Vigo, Spain) in 1996. Previously, in 1989, she joined the Vigo School of Telecom. Eng. as an Assistant Professor, and in 1997, became an Associate Professor, with teaching and research activities in the field of semiconductor devices and circuits for microwave applications. Her main research interests are in the areas of large-signal characterization and modeling of microwave active devices and circuits.

Ahmed Maan ABDULKHALEQ was born in Mosul, Iraq. He received his B.Sc. degree in Communications Engineering (Class Ranking: 1st) from the University of Mosul, Iraq in 2009. In 2013-2017, he worked as a lecturer at the University of Nineveh. Since 2018, he has appointed as an Early-Stage Researcher (ESR)/Design Engineer at SARAS Technology, UK. Currently, he is working as ESR 12 of 13 ESRs of 8 research groups, spread across 4 leading Universities/research institutions and 4 industrial partners in 5 different European countries, targeting a Secure Network Coding for Reduced Energy Next Generation Mobile Small Cells (SECRET) the Horizon 2020 Marie Sklodowska-Curie Action. 